\title{
Nerve root sedimentation sign on MRI: A triage screen for leg dominant symptoms?
}

\author{
Zachary Huschi $^{1} \cdot$ Laura Neuburger $^{1} \cdot$ Syed Uzair Ahmed ${ }^{2} \cdot$ Yanzhao Cheng $^{3} \cdot$ Daryl R. Fourney $^{2}$ (i)
}

Received: 4 March 2021 / Revised: 21 May 2021 / Accepted: 4 July 2021 / Published online: 18 July 2021

(c) The Author(s) 2021

\begin{abstract}
Purpose Surgical indications for lumbar spinal stenosis are controversial, but most agree that leg dominant pain is a better predictor of success after decompression surgery. The objective of this study is to analyze the ability of the Nerve Root Sedimentation Sign (SedSign) on MRI to differentiate leg dominant symptoms from non-specific low back pain.

Methods This was a retrospective review of 367 consecutive patients presenting with back and/or leg pain. Baseline clinical characteristics included Oswestry disability index (ODI), visual analog pain scores, EuroQol Group 5-Dimension Self-Report (EQ5D) and Saskatchewan Spine Pathway Classification (SSPc). Inter- and intra-rater reliability for SedSign was 73\% and $91 \%$, respectively (3 examiners).

Results SedSign was positive in 111 (30.2\%) and negative in 256 (69.8\%) patients. On univariate analysis, a positive SedSign was correlated with age, male sex, several ODI components, EQ5D mobility, cross-sectional area (CSA) of stenosis, anteroposterior diameter of stenosis, and SSPc pattern 4 (intermittent leg dominant pain). On multivariate analysis, SedSign was associated with age, male sex, CSA stenosis and ODI walking distance. Patients with a positive SedSign were more likely to be offered surgery after referral (OR 2.65). The sensitivity and specificity for detecting all types of leg dominant pain were 37.4 and 82.8 , respectively (ppv 77.5\%, npv 43.8\%).

Conclusions Patients with a positive SedSign were more likely to be offered surgery, in particular non-instrumented decompression. The SedSign has high specificity for leg dominant pain, but the sensitivity is poor. As such, its use in triaging appropriate surgical referrals is limited.
\end{abstract}

Keywords Lumbar spinal stenosis $\cdot$ Neurogenic claudication $\cdot$ Nerve root sedimentation sign $\cdot$ Multidisciplinary pathway

\section{Introduction}

Lower back and/or leg pain is a common cause for presentation to primary care. While the large majority of these cases are due to non-specific low back pain and can be handled conservatively, some of these patients suffer from symptomatic lumbar spinal stenosis (LSS) that would benefit from a surgical consultation [1]. Unfortunately, due to its variable

Daryl R. Fourney

daryl.fourney@usask.ca

1 College of Medicine, University of Saskatchewan, Saskatoon, Saskatchewan, Canada

2 Division of Neurosurgery, Royal University Hospital, University of Saskatchewan, Saskatoon, Saskatchewan, Canada

3 Department of Surgery, University of Saskatchewan, Saskatoon, Saskatchewan, Canada presentation, there is controversy with regards to precise clinical and radiological criteria for "symptomatic LSS" and therefore controversy around who requires referrals [2, 3]. Current literature suggests that a substantial portion of referrals to spine surgeons are ultimately deemed not suitable for surgical intervention $[4,5]$. These non-operative referrals create a significant strain on the healthcare system. While various clinical pathways and educational programs have been suggested to improve the efficiency of triage for LSS, there remains significant room for improvement [6-10].

Despite the surgical indications for LSS being controversial, most agree that leg dominant pain is one of the most important clinical predictors for success after decompression surgery [5]. Magnetic Resonance Imaging (MRI) is useful to determine the dural sac cross-sectional area (CSA) and the anterior-posterior (AP) diameter; however, several studies have shown that there is little correlation between these measurements, clinical symptoms, and treatment outcomes 
$[11,12]$. Furthermore, measuring these values typically requires specialized software that is often not available to referring physicians, hindering the application of these measurements as a triaging tool. There is a need to identify easily accessible radiographic signs that can aid in the diagnosis of LSS and assist in triaging the most appropriate referrals to spine surgeons.

Barz et al. introduced the Nerve Root Sedimentation Sign, or SedSign, on MRI as a radiological sign that could aid in the diagnosis of symptomatic LSS [13]. SedSign relies on the tendency of nerve roots to sediment into the dorsal portion of the dural sac due to gravity on supine MRI in the absence of symptomatic LSS (Fig. 1). The original study reported excellent sensitivity and specificity of the SedSign for the diagnosis of symptomatic LSS, although others have had mixed results. This is most likely due to the variable definitions of "symptomatic LSS" present in these studies [14-18]. Additionally, several studies fail to account for leg dominant pain associated with spinal stenosis that does not fit clinical criteria for neurogenic claudication (i.e., sciatica), conditions which likely would still benefit from surgical referral [13, 18, 19].

The aim of this study was to determine the sensitivity and specificity of the SedSign to diagnose neurogenic claudication, sciatica, or leg dominant pain by utilizing a patient population triaged through a multidisciplinary spine pathway that uses standardized criteria for diagnosis by multiple observers. To the best of our knowledge, this is the first study to utilize standardized criteria for the diagnosis of non-specific low back pain, sciatica, or LSS in the analysis of SedSign. Additionally, this is the first study to investigate the utility of SedSign for the diagnosis of all types of leg dominant pain, which has implications for use as a triage tool for surgeon referral.

\section{Materials and methods}

This manuscript has been prepared following the Standards for the Reporting of Diagnostic Accuracy Studies (STARD) guidelines [20]. This study was approved by the University of Saskatchewan ethics board (Bio 18-89). As this was a retrospective review with only aggregate data being presented, individual consent was not required as per ethics review. A retrospective medical record review of prospectively collected data was performed on all consecutive new elective outpatient referrals for lower back and/or leg pain seen by the senior author (DRF) from January 1, 2012, to May 31, 2018, that were triaged to neurosurgical referral by the multidisciplinary Saskatchewan Spine Pathway (SSP). Exclusion criteria included the lack of a SSP classification (SSPc) diagnosis, lack of available MRI, previous lumbar spinal surgery, significant spinal deformity (defined as Cobb's angle $>20$ degrees), spondylolisthesis grade III or higher, significant trauma (defined as fracture), infection, age less than 18 years or greater than 80 years, tumor, cauda equina syndrome, or Worker's Compensation Board claim. As suggested by Barz et al., patients who had lumbar spinal stenosis only at level L5/S1 were also excluded since the S1 and S2 nerve roots exit the dural sac in a ventral position and therefore do not settle, making it misleading or unreliable to interpret the sedimentation sign at these levels (Fig. 2) [13].

The patients' age, sex, smoking status, and date of baseline appointment were recorded. Additionally, the presence of nerve root irritation, as defined by a positive straight leg raise (SLR), and the patient's Oswestry Disability Index (ODI), Visual Analog Scale (VAS) leg, Visual Analog Scale (VAS) back, and EuroQuol 5 Dimension Scale (EQ5D) scores at baseline were recorded.

The SSPc was determined through the multidisciplinary pathway as described by Fourney et al. [8]. The reliability
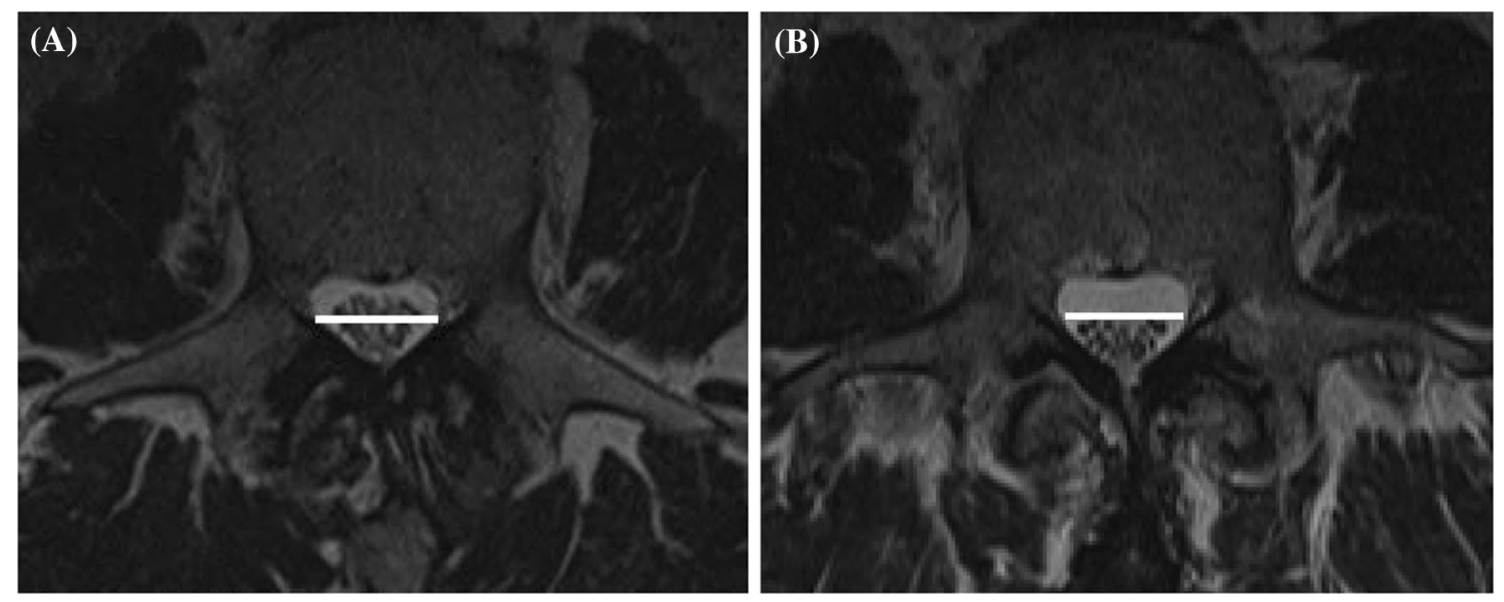

Fig. 1 Positive SedSign a and negative SedSign b on T2-weighted MRI 


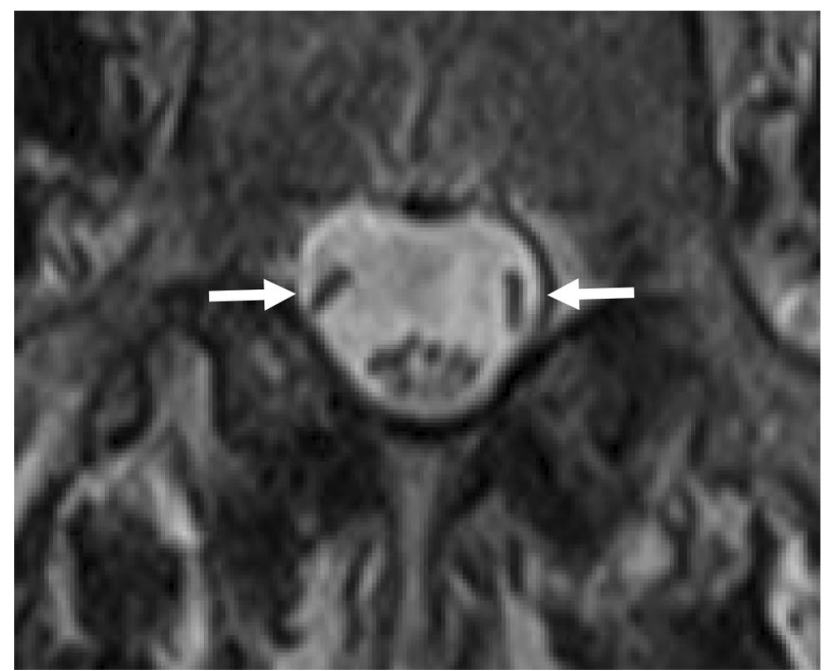

Fig. 2 Axial T2-weighted MRI image showcasing ventral nerve roots exiting the dural sac. These are not included in the analysis of sedsign

and validity of the SSPc have been previously reported [9, 10]. In short, SSPc $1 \& 2$ are back dominant pain patterns. $\mathrm{SSPc} 3$ (sciatica) is leg dominant pain that is constant, associated with positive neurological findings and aggravated by movement. SSPc 4 (neurogenic claudication) is leg dominant pain that is intermittent, aggravated by activity in extension and relieved by rest in flexion. SSPc $1 \& 2$ are mutually exclusive with each other; SSPc $3 \& 4$ can co-exist with each other and/or occur concurrently with SSPc 1 or 2 . In patients with multiple SSPc diagnoses, the first diagnosis is the predominant pattern of pain and this was used for analysis.

Lumbosacral spine MRI scans were accessed using a picture archiving system (PACS) with integrated digital area measurement (Philips IntelliSpace, Inc.). The dural sac cross-sectional area (CSA) and antero-posterior (AP) diameter of the dural sac at each intervertebral disk between L1/2 and L5/S1 were measured by an observer blinded to the SSPc diagnosis. For analysis, radiologic stenosis was defined as a CSA of less than $100 \mathrm{~mm}^{2}$ or an AP diameter of less than $10 \mathrm{~mm}$.

Three raters (ZH, LN, SUA), also blinded to SSPc, evaluated the MR images independently to measure SedSign. According to the protocol by Barz et al., a positive SedSign was defined as the presence of nerve roots in the ventral or central part of the dural sac, while a negative SedSign was defined as all nerve roots being located in the dorsal part of the dural sac, except for the two ventral nerve roots that leave the sac one level below the stenosis (Figs. 1 and 2). Deviating from the original protocol by Barz et al., the SedSign was analyzed at the approximate mid-height of the vertebral body at all available lumbar levels, not just at the levels above and below the maximal stenosis. Other authors have made similar modifications in analyzing the SedSign
[21-23]. A positive SedSign was defined as the presence of nerve roots in the ventral or central part of the dural sac at any level analyzed. SedSign positivity or negativity was determined by the majority of the three raters. In accordance with previous rating protocols, raters were trained using 25 MRI scans to rate SedSign as a group before rating study subjects [15]. To assess intra-observer reliability, the SedSign was re-evaluated by the raters after approximately 6 weeks.

Cohen's $\kappa$ were calculated for the intra-observer and interobserver reliability. A $\chi^{2}$ analysis was performed to see if there was any relationship between SedSign and sex, smoking status, whether or not the patient was offered surgery, and if instrumentation was used. Univariate ANOVA with general linear models was performed on all other patient characteristics. A multivariate analysis using logistic regression was performed for all statistically significant variables found on univariate analysis, with a Hosmer-Lemeshow test as appropriate. SAS programming 9.4 (SAS Institute) was used for all analysis.

\section{Results}

After applying the exclusion criteria (Fig. 3), there were 367 patients (Age $54.7 \pm 14.9$ year; $46.3 \%$ female). A positive SedSign was found in 111 (30.2\%; Age $61.8 \pm 12.7$ year; $33.3 \%$ female), while 256 (69.8\%; Age $51.7 \pm 14.8$ year; $52.0 \%$ female) had a negative SedSign. Patient demographics are displayed in Table 1.

The intra-rater reliability $(\kappa)$ of the SedSign after approximately six weeks was $0.86(0.81-0.91)$. The inter-rater reliability of the SedSign was 0.77 (0.72-0.82).

The sensitivity, specificity, and positive/negative predictive value for the SedSign are shown in Table 2. The sensitivity and specificity for SSPc 4 (intermittent neurogenic claudication) were $50.7 \%$ and $84.4 \%$, respectively. The sensitivity was lower for all types of leg dominant pain (SSPc 3 and 4 combined, 37.4\%) and lowest for SSPc 3 (20.2\%); however, specificity was similar across all groups. Using a modified version of the original criteria for patient selection reported by Macedo et al., in which patient-self-reported walking distance by ODI was used as a surrogate for a treadmill test, the sensitivity and specificity of SedSign increased to $64.2 \%$ and $98.5 \%$, respectively[15].

As shown in Tables 1 and 3, a positive SedSign was significantly correlated with older age, male sex, certain ODI sub-scores (walking, standing), EQ5D mobility, CSA of maximal stenosis, AP diameter of maximal stenosis, SSPc 4 (intermittent leg dominant pain) and all types of leg dominant pain (SSPc 3 and 4 combined). Certain ODI sub-scores (sitting, sleeping), back dominant pain and SSPc 3 (sciatica) were inversely correlated with SedSign (Table 3). Patients 


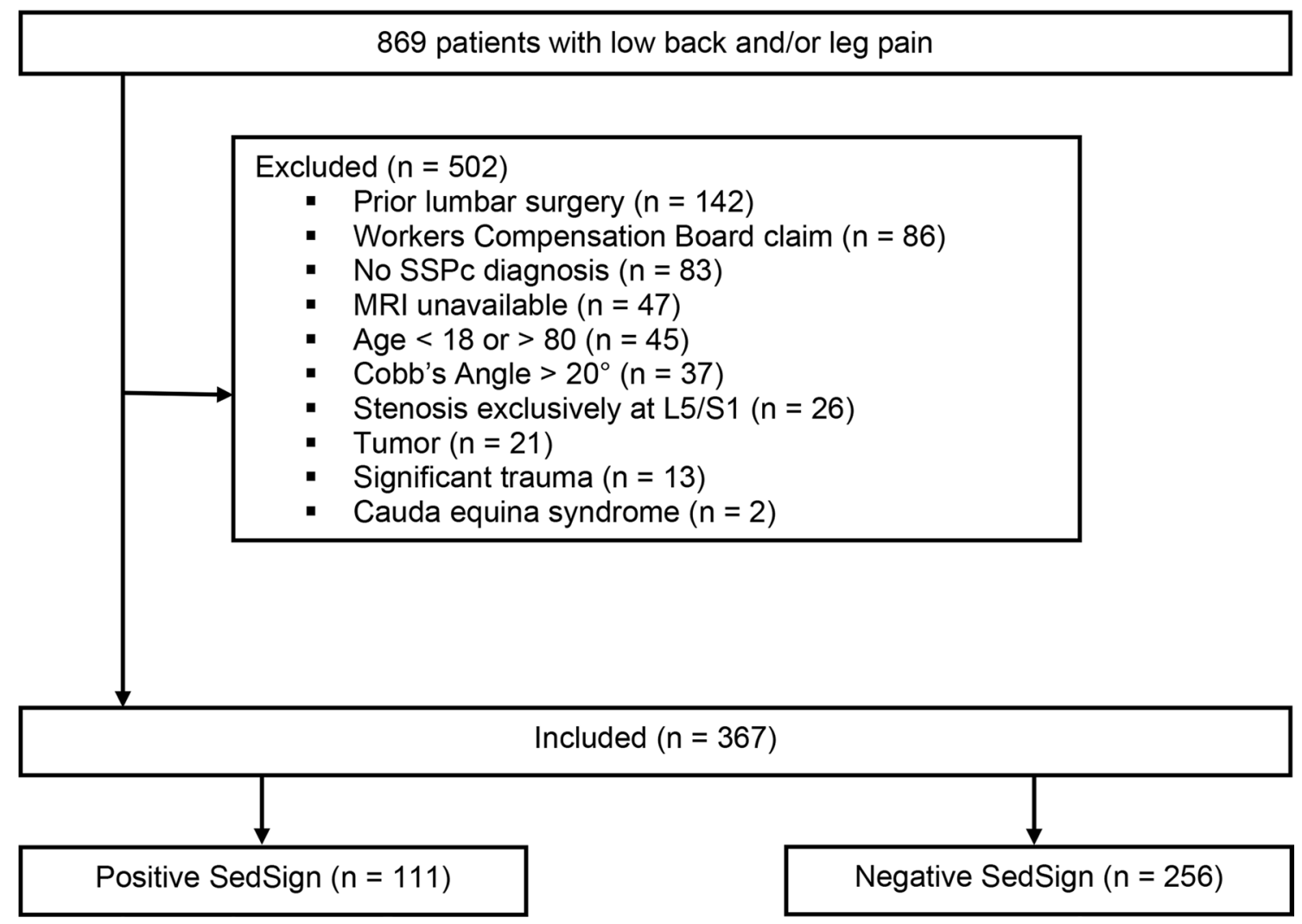

Fig. 3 Study profile of included participants

Table 1 Clinical and radiographic criteria

\begin{tabular}{|c|c|c|c|c|c|}
\hline & Group, $n$ & Sed sign positive & Sed sign negative & Odds ratio & $P$ Value \\
\hline Age in years, mean $\pm S D$ & & $61.78( \pm 12.69)$ & $51.67( \pm 14.83)$ & NA & $<0.0001$ \\
\hline Sex & $\begin{array}{l}\text { Female, } 170 \\
\text { Male, } 197\end{array}$ & $\begin{array}{l}37(10.1 \%) \\
74(20.2 \%)\end{array}$ & $\begin{array}{l}133(36.2 \%) \\
123(33.5 \%)\end{array}$ & NA & 0.001 \\
\hline Smoking status & $\begin{array}{l}\text { No, } 203 \\
\text { Yes, } 148\end{array}$ & $\begin{array}{l}59(16.8 \%) \\
47(13.4 \%)\end{array}$ & $\begin{array}{l}144(41 \%) \\
101(28.8 \%)\end{array}$ & NA & 0.59 \\
\hline $\begin{array}{l}\text { Dural sac CSA at maximal stenosis }\left(\mathrm{mm}^{2}\right) \text {, mean } \pm \\
\text { SD }\end{array}$ & & $59.65( \pm 23.61)$ & $124.8( \pm 55.78)$ & NA & $<0.0001$ \\
\hline $\begin{array}{l}\text { AP spinal canal diameter at maximal stenosis }(\mathrm{mm}) \text {, } \\
\text { mean } \pm \text { SD }\end{array}$ & & $7.15( \pm 1.98)$ & $10.7( \pm 2.77)$ & NA & $<0.0001$ \\
\hline Offered surgery & $\begin{array}{l}\text { No, } 156 \\
\text { Yes, } 207\end{array}$ & $\begin{array}{l}30(8.3 \%) \\
80(22 \%)\end{array}$ & $\begin{array}{l}126(34.7 \%) \\
127(35 \%)\end{array}$ & 2.65 & $<0.0001$ \\
\hline Surgery performed & $\begin{array}{l}\text { No, } 229 \\
\text { Yes, } 138\end{array}$ & $\begin{array}{l}56(15.3 \%) \\
55(15 \%)\end{array}$ & $\begin{array}{l}173(47.1 \%) \\
83(22.6 \%)\end{array}$ & 2.05 & $\mathbf{0 . 0 0 2 0}$ \\
\hline Instrumentation utilized in surgery & $\begin{array}{l}\text { No, } 95 \\
\text { Yes, } 43\end{array}$ & $\begin{array}{l}44(31.9 \%) \\
11(8 \%)\end{array}$ & $\begin{array}{l}51(36.7 \%) \\
32(23.2 \%)\end{array}$ & 0.4 & 0.0212 \\
\hline
\end{tabular}

CSA Cross-sectional area, AP Anteroposterior 
Table 2 Sensitivity and specificity of SedSign for leg dominant pain

\begin{tabular}{|c|c|c|c|c|c|c|c|c|}
\hline & TP & FP & FN & $\mathrm{TN}$ & Sn & $\mathrm{Sp}$ & PPV & NPV \\
\hline SSPc 3 (constant leg dominant pain [sciatica]) & 23 & 24 & 91 & 111 & 20.2 & 82.2 & 48.9 & 55.0 \\
\hline $\begin{array}{l}\text { SSPc } 4 \text { (intermittent leg dominant pain [neurogenic claudica- } \\
\text { tion]) excluding sciatica }\end{array}$ & 70 & 18 & 68 & 97 & 50.7 & 84.4 & 79.6 & 58.9 \\
\hline SSPc 3 or 4 (all leg dominant pain - constant or intermittent) & 86 & 25 & 144 & 112 & 37.4 & 82.8 & 77.5 & 43.8 \\
\hline Modified Barz criteria ${ }^{25}$ & 61 & 1 & 34 & 67 & 64.21 & 98.53 & 98.39 & 65.34 \\
\hline
\end{tabular}

SSPc Saskatchewan spine pathway classification, TP True positive, FP False positive, $F N$ False negative, $T N$ True negative, Sn Sensitivity, Sp Specificity, $P P V$ Positive predictive value, $N P V$ Negative predictive value

Table 3 Patient-self-reported function and Saskatchewan Spine Pathway Classification (SSPc) diagnosis

\begin{tabular}{lllllr}
\hline & Group & Sed sign positive & Sed sign negative & Odds ratio & $P$ value \\
\hline ODI walking, mean \pm SD & & $2.89( \pm 1.24)$ & $2.41( \pm 1.32)$ & 1.33 & $\mathbf{0 . 0 0 2 3}$ \\
ODI sitting, mean \pm SD & & $1.68( \pm 1.32)$ & $2.24( \pm 1.26)$ & 0.71 & $\mathbf{0 . 0 0 0 3}$ \\
ODI standing, mean \pm SD & & $3.23( \pm 1.27)$ & $2.77( \pm 1.37)$ & 1.30 & $\mathbf{0 . 0 0 4 7}$ \\
ODI sleeping, mean $\pm \mathrm{SD}$ & & $1.55( \pm 1.08)$ & $1.92( \pm 1.24)$ & 0.76 & $\mathbf{0 . 0 1 0 7}$ \\
EQ5D mobility & 1 & $8(2.3 \%)$ & $45(13.1 \%)$ & 2.78 & $\mathbf{0 . 0 1 7 8}$ \\
& 2 & $94(27.4 \%)$ & $190(55.4 \%)$ & & \\
Pattern 1 & 3 & $1(0.3 \%)$ & $5(1.5 \%)$ & & \\
& Not pattern 1 & $89(24.3 \%)$ & $156(42.5 \%)$ & 0.39 & $\mathbf{0 . 0 0 0 3}$ \\
Pattern 2 & Pattern 1 & $22(6 \%)$ & $100(27.3 \%)$ & & \\
Pattern 3 & Not pattern 2 & $109(29.7 \%)$ & $252(68.7 \%)$ & 1.16 & 0.99 \\
& Pattern 2 & $2(0.5 \%)$ & $4(1.1 \%)$ & & \\
Pattern 4 & Not pattern 3 & $88(24 \%)$ & $165(45 \%)$ & 0.47 & $\mathbf{0 . 0 1}$ \\
& Pattern 3 & $23(6.3 \%)$ & $91(24.8 \%)$ & & \\
& Not pattern 4 & $48(13.1 \%)$ & $202(55 \%)$ & 4.91 & $<\mathbf{0 . 0 0 0 1}$ \\
\hline
\end{tabular}

$O D I$ Oswestry disability index, EQ5D EuroQol-5D
Table 4 Multivariate analysis

\begin{tabular}{llll}
\hline Odds ratio estimate & & & \\
\hline & Odds ratio & 95\% CI for Odds ratio & $P$ value \\
\hline Sex (male) & 6.73 & $(2.15,21.07)$ & 0.011 \\
$\begin{array}{l}\text { CSA at maximal } \\
\text { stenosis }\end{array}$ & 0.96 & $(0.94,0.97)$ & $<0.0001$ \\
ODI walking & 1.8 & $(1.06,3.05)$ & 0.0151 \\
\hline
\end{tabular}

CSA Cross-sectional area, ODI Oswestry disability index

with a positive SedSign were more likely to be offered surgery - in particular, non-instrumented decompression, as shown in Table 1.

The results of the multivariate analysis are shown in Table 4. Positive SedSign was associated with male sex, CSA of maximal stenosis and ODI walking. For every $1 \mathrm{~mm} 2$ reduction in CSA of maximal stenosis, the odds of SedSign positivity increased $4 \%$. For every one-unit decrease in ODI walking distance, the odds of SedSign positivity decreased $80 \%$.

\section{Discussion}

This study reports the sensitivity and specificity of SedSign for all types of leg dominant pain diagnosed by multiple observers in a standardized, multidisciplinary spine pathway. Similar to others, we found high intra-rater and inter-rater reliability for SedSign [16]. In our cohort, SedSign had high specificity but low sensitivity for the diagnosis of intermittent neurogenic claudication (SSPc 4) and leg dominant pain (SSPc 3 and 4). This study further supports the findings that patient with suspected disk herniations need not to be excluded from application of the SedSign [15]. Additionally, as illustrated in Table 1, patients with a positive SedSign were more likely to receive surgery, in particular non-instrumented decompression.

This is the first study to use a standardized multidisciplinary pathway to aid in the diagnosis of LSS. A more detailed description of the Saskatchewan Spine Pathway (SSP) is published elsewhere; to summarize, patients are seen by multiple providers (primary care, specialized physiotherapist, spine surgeon) who assign a clinical categorization 
based on symptoms and signs [8]. The inter-observer reliability of this classification has been previously reported (agreement $=79 \%, \kappa=0.61$ ) [24]. In the SSP, patients are optimized with non-surgical care before surgical referral. While this may limit the generalizability of the results somewhat, this study benefits from much more clarity of method with respect to patient selection and symptom definition.

In previous studies, SedSign has been associated with a near perfect sensitivity and specificity when used in highly selected patient populations $[13,14,16]$. These studies typically use only one observer or restrict the diagnosis of LSS to those with severe radiologic stenosis. Many studies exclude patients with mild to moderate radiologic stenosis from the analysis entirely. Additionally, in the original study on the SedSign by Barz et al., a treadmill walking test was used to establish the diagnosis of LSS. This study is often not routinely performed at most spine surgery centers.

Due to the limitations of a retrospective review, the results of a treadmill walking test were not available for analysis in our study. In order to compare our patient population with the one present in the original study by Barz et al., we used a modified exclusion criteria proposed by Macedo et al. in which patients ODI walking was used as a surrogate for a treadmill test [19]. Using this method, 204 patients had to be excluded from our calculations due to either their walking distance or their radiologic stenosis falling into an intermediate category which was originally excluded by Barz et al. Eighty six of these 204 (42\%) patients who were excluded using modified Barz criteria ultimately underwent surgery. Therefore, while SedSign may have higher predictive value for symptomatic LSS in the carefully selected patient populations reported by Barz et al., its utility as a screening tool for appropriate surgical candidates in general may be more limited.

We wanted to explore the potential for SedSign to assist practitioners in triaging appropriate surgical referrals. A significant proportion of referrals to spine surgeons turn out to be non-operative, and this creates longer wait times for those patients who require surgery. While the low sensitivity of the SedSign for leg dominant pain limits its ability to act a sole radiologic triage, the high specificity for leg dominant pain can support a higher urgency referral. This may be appealing to spine surgeons, as Table 1 shows that patients with a positive SedSign are more likely to undergo surgery, in particular non-instrumented decompression. Therefore, we believe SedSign should be looked at as adjunct in triaging referrals where surgeons may be most interested in seeing patients with a preponderance of leg dominant pain. This may be especially valuable in centers that employ clinical pathways to assist in the management of low back pain, as a positive SedSign can further reinforce the need for an expedited surgeon referral.
There are several limitations to this study. Firstly, this was a retrospective chart review with the patients derived from a single surgeon's practice; however, the determination of LSS was made through a multidisciplinary triage process (SSP) with well-defined clinical criteria which helps limit the observer bias. Secondly, as this was a retrospective chart review, MR imaging protocols were not standardized, and MRI data was not available for all patients $(47 / 869,5.4 \%)$. Axial cuts were not available for all levels in all patients, and there were variable methods to orient the gantry with respect to the disk space. Although this hinders the standardization of our analysis, this represents the reality for many practitioners who do not have control over these things in their practice. Additionally, studies have shown that morphological parameters tend to be less sensitive to gantry orientation compared to measures of radiologic stenosis like the CSA. As such there may be a benefit to using the SedSign in this scenario where gantry orientation is not standardized [25]. Thirdly, while we showed that patients with a positive SedSign were more likely to undergo surgery in our patient population, we did not collect data on surgical outcomes. Fourthly, not all radiologists report on the presence of the SedSign in their reports and referring providers typically do not review the MRI images themselves. This highlights the need for establishment of standard practices for all, including radiologists, when dealing with low back pain. Finally, this model of assuming that a referral to a spine surgeon is only to determine surgical candidacy downplays the major role of surgeons in assisting or directing non-surgical spine care.

\section{Conclusions}

The SedSign had high inter-rater and intra-rater reliability. It was associated with the severity of stenosis and self-reported walking distance on multivariate analysis. The likelihood of SedSign positivity was inversely proportional to the CSA as a measure of stenosis, and proportional to the ODI walking distance. In patients assessed and referred through a multidisciplinary clinical spine care pathway, SedSign had high specificity for neurogenic claudication and leg dominant pain. Back dominant pain and sciatica were both inversely correlated with SedSign. The sensitivity of SedSign was poor. As such, SedSign should not be used in isolation as a method of "radiologic triage" for spine surgery referrals but may be used as an adjunct to the history and physical exam for determining referral urgency. 
Authors' contributions DRF contributed to the study conception and design. Material preparation and data collection were performed by $\mathrm{ZH}, \mathrm{LN}$ and SUA. Analysis was performed by YC. The first draft of the manuscript was written by $\mathrm{ZH}$ and all authors commented on previous versions of the manuscript. All authors read and approved the final manuscript.

\section{Funding None}

Code availability Not applicable

Data and material availability The datasets generated during and/or analyzed during the current study are available from the corresponding author on reasonable request.

\section{Declarations}

Conflict of interest The author declare that they have no conflict of interest.

Consent to participate As this was a retrospective review with only aggregate data being presented, individual consent was not required as per ethics review.

Consent for publication As this was a retrospective review with only aggregate data being presented, individual consent was not required as per ethics review.

Ethics approval Approved by the University of Saskatchewan Biomedical ethics board (Bio 18-89).

Open Access This article is licensed under a Creative Commons Attribution 4.0 International License, which permits use, sharing, adaptation, distribution and reproduction in any medium or format, as long as you give appropriate credit to the original author(s) and the source, provide a link to the Creative Commons licence, and indicate if changes were made. The images or other third party material in this article are included in the article's Creative Commons licence, unless indicated otherwise in a credit line to the material. If material is not included in the article's Creative Commons licence and your intended use is not permitted by statutory regulation or exceeds the permitted use, you will need to obtain permission directly from the copyright holder. To view a copy of this licence, visit http://creativecommons.org/licenses/by/4.0/.

\section{References}

1. Patel AT, Ogle AA (2000) Diagnosis and management of acute low back pain. Am Fam Physician 61:1779-1786

2. Lohman CM, Tallroth K, Kettunen JA, Lindgren K-A (2006) Comparison of radiologic signs and clinical symptoms of spinal stenosis. Spine (Phila Pa 1976) 31:1834-1840. https://doi.org/10. 1097/01.brs.0000227370.65573.ac

3. Sirvanci M, Bhatia M, Ganiyusufoglu KA et al (2008) Degenerative lumbar spinal stenosis: correlation with oswestry disability index and MR imaging. Eur Spine J 17:679-685. https://doi.org/ 10.1007/s00586-008-0646-5

4. Fourney DR (2010) Waiting lists for lumbar spine referrals in Canada: what is the solution? Can J Neurol Sci 37:719-720

5. Deis N, Findlay JM (2010) Appropriateness of lumbar spine referrals to a neurosurgical service. Can J Neurol Sci 37:843-848. https://doi.org/10.1017/s0317167100051544
6. Akbari A, Mayhew A, Al-Alawi MA et al (2008) Interventions to improve outpatient referrals from primary care to secondary care. Cochrane Database Syst Rev 2008(4). https://doi.org/10. 1002/14651858.CD005471.PUB2

7. Harrington JT, Dopf CA, Chalgren CS (2001) Implementing guidelines for interdisciplinary care of low back pain: a critical role for pre-appointment management of specialty referrals. Jt Comm J Qual Improv 27:651-663. https://doi.org/10.1016/S10703241(01)27055-2

8. Fourney DR, Dettori JR, Hall H et al (2011) A systematic review of clinical pathways for lower back pain and introduction of the Saskatchewan spine pathway. Spine (Phila Pa 1976) 36:S164S171. https://doi.org/10.1097/BRS.0b013e31822ef58f

9. Kindrachuk DR, Fourney DR (2014) Spine surgery referrals redirected through a multidisciplinary care pathway: effects of nonsurgeon triage including MRI utilization. J Neurosurg Spine 20:87-92. https://doi.org/10.3171/2013.10.SPINE13434

10. Wilgenbusch CS, Wu AS, Fourney DR (2014) Triage of spine surgery referrals through a multidisciplinary care pathway. Spine (Phila Pa 1976) 39:S129-S135. https://doi.org/10.1097/BRS. 0000000000000574

11. Kalichman L, Cole R, Kim DH et al (2009) Spinal stenosis prevalence and association with symptoms: the framingham study. Spine J 9:545-550. https://doi.org/10.1016/j.spinee.2009.03.005

12. Jarvik JG, Deyo RA (2002) Diagnostic evaluation of low back pain with emphasis on imaging. Ann Intern Med 137:586-597

13. Barz T, Melloh M, Staub LP et al (2010) Nerve root sedimentation sign: Evaluation of a new radiological sign in lumbar spinal stenosis. Spine (Phila Pa 1976) 35:892-897. https://doi.org/10. 1097/BRS.0b013e3181c7cf4b

14. Kim HJ, Lee KY, Kim WC, Oh YS (2011) Clinical value of nerve root sedimentation sign in lumbar spinal stenosis. J Korean Soc Spine Surg 18:117. https://doi.org/10.4184/jkss.2011.18.3.117

15. Macedo LG, Wang Y, Battié MC (2013) The sedimentation sign for differential diagnosis of lumbar spinal stenosis. Spine (Phila Pa 1976) 38:827-831. https://doi.org/10.1097/BRS.0b013e3182 $7 \mathrm{e} 8 \mathrm{ecd}$

16. Tomkins-Lane CC, Quint DJ, Gabriel S et al (2013) Nerve root sedimentation sign for the diagnosis of lumbar spinal stenosis: reliability, sensitivity, and specificity. Spine (Phila Pa 1976) 38:E1554-60. https://doi.org/10.1097/BRS.0b013e3182a8c2da

17. Laudato PA, Kulik G, Schizas C (2015) Relationship between sedimentation sign and morphological grade in symptomatic lumbar spinal stenosis. Eur Spine J 24:2264-2268. https://doi.org/10. 1007/s00586-015-4021-z

18. Ko S (2018) Correlations between sedimentation sign, dural sac cross-sectional area, and clinical symptoms of degenerative lumbar spinal stenosis. Eur Spine J 27:1623-1628. https://doi.org/10. 1007/s00586-017-5374-2

19. Zhang L, Chen R, Xie P et al (2015) Diagnostic value of the nerve root sedimentation sign, a radiological sign using magnetic resonance imaging, for detecting lumbar spinal stenosis: a metaanalysis. Skeletal Radiol 44:519-527. https://doi.org/10.1007/ s00256-014-2064-6

20. Cohen JF, Korevaar DA, Altman DG et al (2016) STARD 2015 guidelines for reporting diagnostic accuracy studies: explanation and elaboration. BMJ Open 6:e012799. https://doi.org/10.1136/ bmjopen-2016-012799

21. Zhang L, Chen R, Liu B et al (2017) The nerve root sedimentation sign for differential diagnosis of lumbar spinal stenosis: a retrospective, consecutive cohort study. Eur Spine J 26:2512-2519. https://doi.org/10.1007/s00586-016-4435-2

22. Resende VAC, Teixeira A, da Silva JB et al (2013) Lumbar spinal stenosis: sedimentation sign. Coluna/Columna 12:192-195. https://doi.org/10.1590/S1808-18512013000300003 
23. Moses RA, Zhao W, Staub LP et al (2015) Is the sedimentation sign associated with spinal stenosis surgical treatment effect in SPORT? Spine (Phila Pa 1976) 40:129-36. https://doi.org/10. 1097/BRS.0000000000000672

24. Wilson L, Hall H, McIntosh G, Melles T (1999) Intertester reliability of a low back pain classification system. Spine (Phila Pa 1976) 24:248-54
25. Henderson L, Kulik G, Richarme D et al (2012) Is spinal stenosis assessment dependent on slice orientation? a magnetic resonance imaging study. Eur Spine J 21:760-764. https://doi.org/10.1007/ s00586-011-1857-8

Publisher's Note Springer Nature remains neutral with regard to jurisdictional claims in published maps and institutional affiliations. 